\title{
LA ORTOGRAFÍA ACENTUAL EN LA LENGUA MISKITU: ENRIQUECIMIENTO DE LA EDUCACIÓN INTERCULTURAL BILINGÜE
}

\author{
Ruby Morales Quant ${ }^{5}$ \\ Marcos Padilla Brown ${ }^{6}$ \\ MSc. Yuri Zapata Webb
}

\begin{abstract}
RESUMEN
$\mathrm{E}$ 1 estudio se realizó en diez comunidades: cinco del Wangki, municipio de Waspam y en localidades de hablantes Tawira de Puerto Cabezas. Participaron un total de 109 personas entre hombres y mujeres. Su propósito fue demostrar que la acentuación es pluri-
\end{abstract} fonética y no monofonética, como se ha estado considerando hasta este momento. A pesar de que la ortografía acentual del Idioma Español no tiene concordancia lingüística ni gramatical con la del Mískitu, ha sido la base referencial para el estudio, lo cual permitió hacer adaptaciones al sistema de acentuación de la lengua en cuestión.

La metodología es cualitativa y participativa en la que se aplicaron entrevistas, diálogo, asamblea, cultos de oración, programas radiales y consultas bibliográficas. Como la mayoría de las fuentes no saben leer se utilizaron láminas y objetos concretos. Este estudio, además de servirle a los docentes de educación bilingüe, les será de mucha utilidad a investigadores de la cultura de pueblos indígenas como la del miskitu, a traductores del español al idioma miskitu o viceversa, porque además de la acentuación se le brinda información sobre el uso correcto del circunflejo, que en muchos escritos lo han aplicado incorrectamente.

5 Lic. en E.I.B. y en CCEE con mención en Historia. URACCAN. Bilwi. RAAN. Nic.

6 Lic. en E. I. B. URACCAN.

7 Máster en estudios Interdisciplinarios. URACCAN. Bilwi. RAAN. Nic. Tutor de la investigación. 


\section{INTRODUCCIÓN}

El presente trabajo monográfico titulado La Ortografía Acentual en la Lengua Miskitu, Enriquecimiento de la Educación Intercultural Bilingüe, se realizó para alcanzar tres objetivos: analizar la acentuación de las palabras de estructuras simple y compuesta, identificar los elementos lingüísticos que influyen en la determinación del acento fonético en las palabras de estructuras simple y compuesta, y demostrar que la ortografía acentual en la lengua Miskitu tiene dos sílabas tónicas.

Como docentes miskitos conocedores del idioma Mískitu, una de las razones de hacer este estudio es que hemos tenido inquietudes, preocupaciones con respecto a la implementación del Sistema Educativo Autonómico Regional (SEAR), que promueve la enseñanza de calidad en todos los niveles desde la Educación Preescolar hasta las Universidades. Otra razón es que se carece de información sobre la acentuación en el idioma Mískitu. A pesar de que se han publicado varios libros de la gramática de esta lengua en ninguno se habla con fundamentación sólida sobre el de tema de ortografía acentual.

El lenguaje escrito del idioma Miskitu fue introducido por los misioneros de la Iglesia Morava cuando el segundo contingente de ellos se hizo presente en el territorio llamado La Moskitia, por el año mil novecientos dieciséis. Fueron los primeros en publicar la gramática miskitu y el diccionario de esta lengua vernácula, elaboraron el primer material didáctico para alfabetizar a líderes miskitu para que le apoyaran en la tarea de evangelización, estrategia que le resultó muy fructífera.

La Iglesia Católica publicó el primer diccionario trilingüe: miskitu, español e inglés, este documento es una de las obras más completa que se conoce hasta este momento, pero en cuanto al acento prosódico, se alinea con el autor de la gramática morava.

Los textos de matemáticas y lengua materna que fueron elaborados para la gran cruzada nacional de alfabetización estaban acordes con la realidad del miskitu, aunque los contenidos semánticos y los gramaticales fueron débiles.

La "Ortografía Acentual”, es un tema que no ha sido estudiado por ninguno de los investigadores que han publicado la gramática miskitu; tampoco ha sido incorporado como contenido en los programas de Educación Intercultural Bilingüe (EIB), específicamente en los de la lengua materna mískitu. Esta fue una de las razones que nos impulso a realizar el estudio.

La acentuación del idioma mískitu está siendo influenciada por la ortología española; esta situación se ejemplifica con la palabra "miskitu", cuyo acento prosódico original lo lleva en la primera sílaba (mis), pero muchos hablantes acentúan en la segunda sílaba (ki). Este fenómeno lingüístico va incrementándose por no disponer de normativa de la ortografía acentual. 
El patrón de la ortografía acentual del mískitu es diferente al del español, por ejemplo en español parte de la última sílaba hacia la primera, mientras que en el mískitu, es todo lo contrario, se inicia de izquierda hacia la derecha afectando las dos primeras silabas nada más; en cambio el español afecta la última, penúltima, antepenúltima y la anterior a la antepenúltima sílaba, iniciando de derecha a izquierda.

Los autores que han publicado libros de gramática miskitu afirman que el acento prosódico de ésta lengua va solamente en la primera sílaba; este criterio lo sitúa en monofonético, característica que carece de fundamentación científica. El criterio anterior lo contrastan por los dueños de la lengua, porque además de llevar la fuerza de voz en la primera sílaba tonal existe igual cantidad de vocablos que acentúan en la segunda sílaba tónica.

Este estudio enriquecerá la Educación Intercultural Bilingüe (EIB), específicamente a los docentes y los estudiantes de la escuela primaria que desarrollan los programas y reciben las clases en lengua materna miskitu. Servirá como material bibliográfico a los escritores, investigadores y traductores que se dedican al estudio de la lengua miskitu. Este trabajo viene a llenar los vacíos que existen en los textos de la gramática mískitu y enriquecerá los contenidos programáticos de la Educación Primaria.

\section{REVISIÓN DE LITERATURA}

La literatura sobre la temática abordada en este estudio es poca, pero ayudó a darle soporte científico.

\section{ORIGEN DE LOS INDIOS MISKITOS}

El origen exacto es desconocido. Hay dos posibles teorías respecto al origen de los indios miskitus. Algunos creen que vinieron de la cuenca amazónica. Otros, como Adolfo Vaughan (un indio miskitu, habiendo hecho una investigación del problema) propuso que vinieron del extremo de Honduras. Según una leyenda de los antepasados los miskitus vinieron de aquella región situada al oeste de la laguna de Caratasca. Dicha región se encuentra en el punto donde los ríos Patuca y Coco se aproximan (Wilson John, 1990:42).

En realidad nadie habla una lengua pura; todos hablamos una variedad lingüística. La diferencia que comúnmente se concibe entre lengua y dialecto es política más que lingüística (Moreno Cabrera, 2000:.47).

El conjunto ordenado de sonidos del idioma se denomina Alfabeto Fonológico; la fonología es la parte de la gramática que estudia los sonidos del idioma: su producción, 
su articulación, sus combinaciones, su entonación, etc., y la fonética enseña a pronunciar correctamente las sílabas y las palabras, (Peńa Hernández, 1991:158).

\section{El Alfabeto Miskitu}

He aquí el orden de los veinte sonidos que se compone el alfabeto fonológico del idioma miskitu: a, â, b, d, g, h, i, ì, k, l, m, n, p, r, p, s, t, u, û, w, y. Las vocales del idioma miskitu son seis, tres cortas $(\mathrm{a}, \mathrm{i}, \mathrm{u})$ y tres largas $(\hat{\mathrm{a}}, \hat{\mathrm{i}}, \hat{\mathrm{u}})$, tradicionalmente a éstas tres últimas se han escrito con un acento circunflejo (Salamanca Danilo, 2000:20).

Las vocales cortas tienen la característica de constituirse en sílabas, ellas son las que representan a la clasificación silábica de monolítera. La función lingüística es formar sílabas por sí solas o asociándose con las consonantes.

\section{El diptongo en la primera sílaba tónica}

-ai: baila, daiwan, laila, maisa, naikra, paita, raiti, saiwan, tairi, waitna.

-ia: biara, kiama, piatu, siaya, tiara, piawan, daukaia, kaubaia,

\section{El diptongo en la segunda sílaba tónica}

-ai: alai.

-ia:dukia, klukia, lakia, mukia, lupia, sikia, kupia, lipiatung.

-au: birau, kasau, mapau, dadaukra, kakauhbra, tataukra, kakauka, nanaukra.

-ua: sipuas.

En el Miskitu el triptongo se incorpora solamente en la primera sílaba tónica y se caracteriza por producirse con sílabas de clasificación inversa y mixta. Las combinaciones que presentan son: iai, iay, iau, uai, iaw, uaw, uiw. A continuación, los ejemplos:

-iai: biaira, kiaima, kiaimka, kiaimalula, tiailka

-iay: kiayka, siayka.

-iau: kiaubaia.

\section{Consonantes del Miskitu:}

En el idioma Miskitu hay catorce consonantes que son: b, d, g, h, k, l, m, n, p, r, s, t, w, y. Estos dos últimos fonemas consonantes ( $w, y)$ son considerados semivocales, porque emiten sonidos vocálicos de $\mathbf{u}$ y de i, respectivamente. Estas consonantes tienen tres no- 
taciones que son: según el punto de articulación, según el modo de articulación y según la sonoridad (Urbina Miguel, 2004: 20).

La consonante ka admite una sola combinación que es ks; los ejemplos son: aibuks, aimaks, 765 aisaks, aibliks, ailaks, aikaiks. La consonante ele se combina con $\mathbf{k}, \mathbf{p}$, s, y se constituye la primera coda silábica compuesta, así: - lk: bilkkira, - lp: silp - ls: aipuls . La consonante eme se combina solamente con la ese en la que ella se destaca como primera coda silábica compuesta, así: aipams, aisams,

La consonante ene se combina con la ge y ese en la que resulta ser la primera coda silábica compuesta. A continuación se presentan los ejemplos:

- ng: tibang, asang, bangbang, butsung

- ngks: ailangks, ilingks.

- ns: aiwins, sinska.

Para Castell B. 1995: 7), la sílaba tónica: es la sílaba que se pronuncia con mayor fuerza de voz dentro de una palabra; cada palabra tiene una sílaba tónica y su acentuación a veces se indica por una tilde o acento ortográfico. Según el Océano Uno, la sílaba tónica es la mayor intensidad con que se pronuncia una sílaba de una palabra (Diccionario Enciclopédico Ilustrado, 1992).

\section{Materiales y MÉTODOS}

El tipo de estudio es descriptivo, con enfoque lingüístico-gramatical en donde se describe las características de la ortografía acentual de la lengua miskitu, tema que no ha sido profundizado, debido a ello existe mucho escepticismo en los estudiosos de la lengua, aún en los propios hablantes del idioma. El universo lo constituyeron diez comunidades, cinco de origen tawira del municipio de Puerto Cabezas que son: Krukira, Tuapi, Kamla, Lahmlaya, Yulu y Bilwi (los barrios Spanistown, Nueva Jerusalén y Loma Verde). Y cinco del sector Wangki: Asang, Wiwinak, Andris, Tasbapain y Saupuka.

En cada una de las comunidades se entrevistó a 8 personas como promedio (entre hombres y mujeres) con la excepción de Asang, además de los ocho comunitarios y comunitarias se aprovechó a los 35 estudiantes (30 varones y 5 mujeres) de URACCAN que se están formando para Técnico Superior en Educación Intercultural Bilingüe. 


\section{CoMo TÉCNICAS SE UTILIZARON:}

- La entrevista individual se aplicó a las ancianas y ancianos en las comunidades de tawira, en los barrios de la ciudad de Bilwi y en las localidades del sector wangki, cuyo producto nos resultó muy enriquecedor para la investigacion.

- El diálogo se desarrolló con los sabios ancianos y las sabias ancianas de las comunidades, fueron fructíferos por la abundante y valiosa información que se recabó para el estudio.

- Los programas radiales que se desarrollan en las emisoras locales de Bilwi y Waspam, fueron los medios positivos para verificar la pronunciación y la acentuación de las palabras.

- Los cultos religiosos que celebran en las iglesias cristianas de diferentes denominaciones, fueron aprovechados para detectar la acentuación y la pronunciación de algunas palabras que presentaron dudas.

- Las reuniones y las asambleas comunales en las que presenciamos fueron las mejores técnicas para recabar las informaciones programadas en el estudio.

- Los libros de consulta fueron las fuentes de mayor información para el estudio; a pesar de que en su mayoría eran de la gramática española, los conceptos y las definiciones fueron las brújulas que marcaron el norte en las adaptaciones de los temas del estudio.

- El uso de láminas o dibujos y objetos concretos (hojas, palmas, frutas) dieron los resultados positivos en las entrevistas con las personas analfabetas.

Criterio de selección que se usó para la selección del área de estudio se basó en la cantidad poblacional, por ser las más numerosas entre las comunidades de la Región Autónoma del Atlántico Norte (RAAN). Un segundo criterio fue por considerarlas como comunidades históricas de las Iglesias Morava y Católica; en esas comunas las dos entidades religiosas iniciaron la misión evangelizadora del cristianismo. El tercer y último criterio fue, el índice de analfabetismo que en éstas son menos marcados que en las otras comunidades miskitu de los municipios de Puerto Cabezas y Waspam. 


\section{OPERACIONALIZACIÓN DE VARIABLES}

En cumplimiento de los objetivos propuestos para el estudio de la ortografía acentual en la lengua miskitu, se determinó como variables del estudio a: acento prosódico, sílabas prolongadas, homógrafa, sílaba inversa, sílaba mixta, diptongo, triptongo e influencia de la consonante hache

\section{RESULTADOS Y DISCUSION}

\section{OrTOGRAFía ACENTUAL DEL MISKITU}

La ortografía acentual del idioma miskitu es regida por un sistema de acentuación bifonética en el que afectan a las dos primeras sílabas de la palabra, a las cuales las denominan sílaba tónica o prosódica, también le llaman fonética. Al hacer una comparación con la ortografía acentual española se nota el gran contraste que existe. En la del miskitu inicia de izquierda a derecha, tomando en cuenta la primera y la segunda sílaba tonal, en la del castellano es todo lo contrario, comienza de la derecha hacia la izquierda afectando la última, penúltima, antepenúltima y anterior a la antepenúltima sílaba.

\section{ACENTO PROSÓDICO DEL MISKITU}

Sobre el acento prosódico de las palabras del idioma miskitu los autores que publicaron la gramática miskitu mantienen el mismo criterio de la primera que publicaron los moravos en el año 1927 A continuación se presentan las opiniones de estos investigadores:

Salamanca D. ( 2000:23) expresa así: El acento prosódico en las palabras miskitu recae generalmente en la primera sílaba. Si dejamos de lado algunas palabras compuestas o formadas con prefijos, hay solo unas cuantas excepciones; las más frecuentemente mencionadas son: aisabé (hasta la vista, derivado de aisabia: hablaremos), umpira( pobre) y sakuna( pero-que es en realidad una palabra compuesta: sa-kuna). En cambio Norwood S. (1993:25) sostiene que el acento prosódico en miskito recae prácticamente siempre en la primera sílaba de la palabra.

Urbina M. (2004:35) afirma: El idioma miskitu casi siempre las palabras reciben o llevan la mayor fuerza de voz en la primera sílaba, aunque ortográficamente no se acentúan. Al respecto, Martínez E. (1995: 10) plantea: En miskitu no hay acento prosódico. El fonético recae sobre la primera sílaba, con escasa excepción como aisabé (adiós), que se pronuncia aisabé. En las palabras tomadas de otros idiomas el acento pasa a la primera sílaba. Y Adolfo V. (1995:10) afirma: El acento en el idioma miskitu siempre cae en la primera sílaba. 
Todos los autores de la gramática miskitu coinciden que el acento prosódico de las palabras del idioma es monofonético o sea que tiene una sola sílaba fonética que es la primera. Los hablantes tawira como los wangki han demostrado que además de llevarla en la primera tonal existe otras palabras que acentúan en la segunda sílaba tónica, las cuales pertenecen a la segunda categoría acentual.

El autor de la Gramática Escolar del Miskitu, sostiene que las palabras umpira y sakuna son las dos únicas que pertenecen a la segunda categoría acentual lo cual solo sakuna pertenece a la segunda sílaba tónica y umpira, a la primera por la influencia del sonido um que es sílaba inversa. \}

En cuanto a la palabra aisabé, la autora del libro Introducción al Estudio de la Lengua Miskitu afirma que es la única exceptuación. En primer lugar, se escribe con la vocal i al final (aisabi); en segundo lugar, en el sistema de acentuación del miskitu admite solamente dos sílabas tónicas, y por último, este vocablo lleva el acento prosódico en la primera sílaba tonal por la influencia del prefijo ai.

\section{ELEMENTOS LINGÜÍSTICOS QUE DETERMINAN EL ACENTO PROSÓDICO}

Las vocales largas $(\hat{\mathbf{a}}, \grave{\mathbf{i}}, \hat{\mathbf{u}})$ influyen en la determinación del acento prosódico las cuales producen sonidos prolongados al incorporarse en las sílabas de clasificación inversa y mixta. Estas aparecen solamente en la primera sílaba tónica en la cual recae la fuerza de voz.

El prefijo reflexivo ai es propio de los verbos reflexivos; en él recae la fuerza de voz y en todas las formas verbales conjugadas. Como ejemplo tomamos al verbo aiwaia y se presentan las formas conjugadas:

- aiwanisna, aiwanisma, aiwanisa (Presente Indefinido);

- aiwanuna, aiwanuma, aiwanuya ( Presente Absoluto);

- aiwanri, aiwanram, aiwanan (Pretérito Indefinido);

- aiwanatna, aiwanatma, aiwanata ( Pretérito Absoluto);

- aiwanamna; aiwanma, aiwanbia (Futuro Indefinido).

-aiwanaisna, aiwanaisma, aiwanaisa (Futuro Absoluto);

- aiwanaina, aiwanaima, aiwanaiya (Condicional Tawira);

-aiwanawana, aiwanawama, aiwanawaya (Condicional Wangki); 
El triptongo es elemento muy propio de la primera categoría acentual, porque solo en esa posición se produce para ejercer la fuerza de voz. Las combinaciones vocálicas que concurren son: iai, iau, uai, iaw, aiw, uaw, uiw, iay.

La sílaba inversa, igual que las vocales largas y el sufijo reflexivo solamente se ubica en primera sílaba para determinar el acento prosódico. Esta domina a la sílaba directa y a la mixta o sea ella es la que determina la fuerza de voz cuando éstas se ubican en la segunda sílaba tonal. Por ejemplo en las palabras alba e ispan, el acento fonético se ubicó en la primera sílaba por la influencia de la inversa.

Las homógrafas: Son pares de palabras que la mayoría son bisílabas; el acento fonético de éstas se reparten entre ambas sílabas tónicas, lo cual presenta dificultad en determinarlo a simple vista, por ejemplo la pareja de palabra wina (carne o cuerpo) y wina (de o desde) sin los significantes o significados sería difícil cual de ellas lleva la fuerza de voz en la primera o en la segunda sílaba tónica, para superar este fenómeno se le aplicó la tilde al segundo vocablo o sea la que recibe el acento prosódico en la última categoría acentual, así wina y winá.

La letra hache: La letra $\mathbf{h}$ aparece en la coda silábica de la inicial y en la segunda sílaba se ubica en la cabeza y en la coda. En las palabras prestadas tales como aspital (hospital), asa (hacha) e ilpa (helper) se omitió la letra hache al miskitizarlas, pero el acento fonético se mantiene en la primera silaba tónica por la influencia de la inversa.

Se comprobó que las palabras que se tomaron prestadas del inglés y del español que llevan el fonema $\mathbf{h}$ en la inicial como hospital durante el proceso de miskitización se omiten, esto significa que no es correcto llevar en los escritos este grafema ya que los hablantes tanto los wangki como los tawira no la pronuncian.

La sílaba mixta: La mixta es la sílaba de estructura completa por lo que llevan cabeza, cima y coda silábica. Esta se ubica en la inicial y en la final o sea en la segunda categoría fonética para determinar la fuerza de voz. Ante la inversa se doblega pero frente a la sílaba directa no, ella se impone llevando la fuerza de voz como en las siguientes palabras: ansar (inversa dominando a la mixta), yabal y bulni (la mixta imponiendo a sílaba directa).

El diptongo: El diptongo es cima compuesta de dos vocales y hace su aparición en las dos sílabas tónicas (primera y segunda); las combinaciones son las siguientes: ai, ia, au, ua, iu, ui, aw, iw y ay. Cuando éstas se ubican en la primera o en la segunda sílaba tónica la fuerza de voz recae en ella.

El morfema derivativo ay, por ser prefijos se ubica en la primera sílaba tónica llevándose consigo la fuerza de voz, así: ayapra, ayikra y ayurbra. Son sustantivos parasíntesis que lo construyen con los verbos cuya silaba inicial es una monolítera como apaia, ikaia, ulalia o bien puede que sea una sílaba inversa como alkaia, irbaia, ulbaia.

La mayoría de las palabras que admiten la combinación ai son sustantivos conjugados los cuales le corresponden al segundo poseedor, así: taiwa (mi pelo), maiwan ( mi 
cara), naikra ( mi ojo),laila (mi cabeza), kairma ( mi garganta). Las combinaciones aw, iw, uw entran como diptongo, porque la doblevé funciona como vocal o (ao, io, uo.), debido a este fenómeno los lingüistas consideran como semivocal.

Igual que la consonante anterior la ye es semivocal, porque emite el sonido de la vocal e en la combinación ay que se pronuncia ae. Son sus ejemplos: tayka, mayka, tayira, layira y playka. Esta no tiene la capacidad de asociarse con el resto de las vocales (i, u).

Todas las combinaciones del diptongo presentadas en el primer acápite entran en la primera sílaba tónica, pero en la segunda, solamente cinco de ellas se ubican para determinar la sílaba fonética y son éstas: ia (kupia, lakia, lipiatung), ai (alai, briaisa, piaisa, dimaisa), au (dadaukra, tataukra, krakraukra), aw (lalawra, tatawra, papawra) e iw (krakriwra, srasriwra, srasriwra).

El diptongo es dominante ante la silaba directa, por ejemplo los vocablos tiara y lipiatung llevan diptongo tanto en la inicial como en la segunda y la fuerza de voz está en las sílabas diptongadas tia y pia. Este elemento lingüístico aparece en las dos sílabas tónicas.

Palabras derivadas: La acentuación de las derivadas es heredada por la palabra progenitora; si ésta es de la primera acentuación todas las que surgen de ella llevarán la fuerza de voz en esa categoría acentual, por ejemplo: Los vocablos antika, antikira, antilpia y antitara acentúan en la inicial, porque derivan de la palabra madre anti. Igual ocurre con las primitivas de acentuación en la sílaba posterior a la primera, así: mayas, mayira, mayalpia y mayi, cuya progenitora es mayá.

Palabras parasintéticas: Estas son palabras que se derivan de un verbo, a la raíz de éste se le antepone el prefijo que nace de la sílaba inicial y se le pospone el sufijo ra, el cual es invariable. La acentuación de las parasintéticas se presenta las características siguientes: acentúan al prefijo cuando el progenitor es un verbo reflexivo, así: aiwawanra, del verbo aiwanaia; aiklaklabra de aiklabaia y aisasara de aisaia. La parasintética cuyas sílabas tónicas están constituidas de silabas directas, si el verbo progenitor es de acentuación inicial o es de la segunda tónica, el acento prosódico de ésta se mantiene del progenitor, como en estos ejemplos: dadira de diaia y mamakra de makaia.

Palabras compuestas: Las compuestas se construyen con dos palabras de estructura simples o sea que tiene dos lexemas. La acentuación de éstas durante el proceso de composición no toma en cuenta el acento fonético del segundo vocablo (así: maka y dusa, ambas se acentúan en la sílaba inicial y al unificarlas resultó makadusa. Si la primera palabra es acentuada en la inicial o en la segunda, esa sílaba tónica se conserva en todo el proceso de composición. 
ACENTUaCión de laS Palabras de eStructura SIMPle

Las bisílabas simples

En el léxico del idioma miskitu, las palabras bisílabas son las que representan la mayor cantidad, pero en cuanto la acentuación se reparte entre ambas sílabas tónicas.

He aquí los ejemplos de las palabras que llevan la fuerza de voz en la primera sílaba tonal:

- baku, bila, bukra, buksa, basni, bitni, bangbang, bulni;

- dama, diran, duri, damni, disang, dusa, dutki ;

- bahkia, dahra, klahkla, mahta, nahki, pahni, rahra, sahkal, tahti, wahri, yahma;

- kara, kiru, kuri, krusa, klakla, krabu, krikri, krisi;

- lama, limi, lula, lamni, langni, lasni,lapta, lasa;

- mani, mina, mula, musa, mulka, misla, masa, manka;

- nari, nika, nuka, naka, nasma, nusa, nitka, nânka, ninka;

- pana, pira, punu, pupu, pusal, putka, plasni, pruwra;

- sari, sika, sumi, supa, sunu, slula, slabla, sûtki, sâtka

- tala, trisu, tulu, tuba, tulu, tusban, tûtni, tuhtu, tahpla, tahplu;

- wasi wiwi, witni, wasla, wangki, wina, wingku, wapni, warwra;

- yami, yulu, yula, yasku, yamni, yakwra,

A continuación los ejemplos de las palabras bisílabas que aceptan la fuerza d voz en la segunda sílaba tónica:

- wabul, saba, tabu, tuba, mibi, labu, bara, bisi, blisin, subil, tibang, libang;

- badu, dudul, badi, lida, sadik o sidik;

- bihu, kuhua, lalah, laha, muhu, naha, rahua, waha;

- maka, mika, muku, traka, sika, saka, suka, takru sukia, dukia, lakia, ikia;

- bulit, paplit, pulis, pilar

- tama, mama, tuman, pramis, saman, maman, rami, sumu;

- nani, nina, dunu, dinar, kunin,

- krupia, ,nipil, papa; sapa, supa

- bara, tara, diara, wari,yari, ; 
- pasis, pusa, pisa, sisin, asang, misar;

- tati, mutur, butar, bitar;

- tawa, tawan, yuwa, yawan;

- ayan, taya, maya;

\section{Las trisílabas simples}

Las palabras trisílabas de estructura simple ocupan el segundo lugar en cuanto a cantidad se refiere y en su mayoría son verbos. En cuanto a la acentuación, se distribuye entre las dos sílabas tónicas.

A continuación se presentan los ejemplos de vocablos trisílabos que acentúan en la sílaba inicial de la palabra:

-awala, apaia, arari, âsmala, âspital, ânsara, alwani o alwanai;

- ispara, isbaia, irbaia, impiara, itbaia, istapla, imyula;

- umpira, ulbaia, utbaia, uhrwaia, uskaika, usbaia;

- bisbaya, bulwaia, buskaia, burbaia, bulkaia, blikaia;

- drabaia, daskaia, dipasta, dakaia, dukaia, dribaia;

- kikaia, kulkaia, kiskaia, kutbaia, kihrbaia, kluhngbaia,

- lawana, laswaia, lisbaia, lakaia, lihkaia, lakula;

- masaya, makaia, miskaia, miskitu, mustukra;

- nandusa, ninkara, namika, nanara, nikbaia, nukaia;

- plikaia, palaia, priskaia, paskaia, pindarka, puskaia, putmaya;

- rakaia, rutkaia, ruhngkaia, raskaia, rihngbaia;

- srikaia, srukaia, srutkaia, sutkaia, srikbaia, sipkaia;

- tahbaia, tuhbaia;

- walaia, wasbaia, wisbaia, wilkaia, wistiting, wailangkra, waisaku;

- yamukla, yabaia, yukaia, yuskaia, yahbaia;

En el listado siguiente, las trisílabas simples lleva el acento prosódico en la segunda sílaba tónica:

- babatni, babikiu, tuburus, yabala; 
- mukula, mukurus, likaka, rikaya, maklala, kakamuk, mukuring, mukuring, sakuna;

- pulaia, pilala, ilili, lilura, kalila;

- mamaia, dimaia, samaia, sumura, klamaia, srimaia, lamaia, umukus, tumaia;

- inaia, winaia, sunaia, munaia;

- plapaia, wapaia, wipaia, sipaia, sripaia, lalalni, kupuhlni, lalahni, sapaia, klipaia, suapaia;

- urus;

- basala, usupum, kisura, lisamra, busukra;

- batana,

\section{Las cuatrisílabas simples}

Las cuatrisílabas de estructura simple son muy limitadas y en su mayoría son verbos en infinitivo; éstas presentan las mismas características de las dos primeras clasificaciones. El acento fonético es igual que en las bisílabas y trisílabas o sea que unas llevan la fuerza de voz en la primera sílaba tónica y otras, en la segunda.

Ejemplo:

- aiklabaia, aiwanaia, aipamaia, aismamaia, aisubaia, aimakaia;

- iskadura, implikaia,

- urtakaia, unsabaia,

- kahmkabaira, kuskuspiram, kuktakaia;

- latawira,

- nutakaia,

-rutakaia

Los ejemplos de las cuatrisílabas que llevan el acento prosódico en la segunda categoría acentual son éstas:

- abakaia, abalkaia, isingbaia, ubulkaia,

- sukulnana, sikibrantu,

- pilipita,

- kumadura, rumatutu;

- tininiska, 
- wipusiana,

- puramaira

- susumaya

- patitara,

\section{Acentuación de las palabras de estructura compuesta}

Las palabras de estructura compuesta se caracterizan por constituirse de dos lexemas en algunas, y en otras una raíz con dos morfemas. Entran es este grupo las palabras derivadas, parasintéticas y compuestas.

\section{Las derivadas}

Las derivadas son palabras constituidas por un lexema y un morfema; este último elemento bien pueden ser sufijos diminutivos, aumentativos, posesivos, etc. La acentuación de estos vocablos es heredada de su progenitora, de manera que si ésta lleva el acento tonal en la primera o en la segunda sílaba tonal, todas lo mantienen el acento fonético de la primitiva.

En los ejemplos que a continuación se presentan, la acentuación de la palabra primitiva o la progenitora está sombreada, además llevan tilde las de la segunda categoría acentual:

- dama: damiki, damikam, damika, damikas, damikira, damalpia, damikira;

- aisa: aisalpia, aisikas, aisikira, aisiki, aisikam, aisika;

- masa: masalpia, masika, masiki, masikam, masatara;

- krusa: krusi, krusam, krusas;

- patá: patí, patám, patas;

- saká: sakí, sakám, sakálpia, sakábila, sakátara.

- kisí: kisílupia, kisítara;

- batána: batánka, batánira, batánkas;

\section{Las parasintéticas}

Las parasintéticas del idioma español se construyen de un vocablo compuesto con un derivado, como: sordomudez, en cambio las del miskitu lo realiza con la raíz del verbo aplicando la siguiente regla: prefijo + raíz del verbo + el sufijo ra. 
Tomamos al verbo smalkaia como modelo, la sílaba inicial de éste es smal (es una mixta), la convierte en sílaba directa para obtener el prefijo, así: sma; éste se le antepone a la raíz de smalkaia (smalk) y después se le pospone el sufijo ra (sma+smalk+ra). La parasintética que resultó es smasmalkra.

El acento prosódico de las parasintéticas puede estar en la primera sílaba tónica o bien en la segunda, según la influencia de elementos lingüísticos que intervienen a determinar la acentuación. Por ejemplo, en smasmalkra la fuerza de voz recae en la smal, porque la primera (sma) es una directa y es débil ante la mixta.

Las parasintéticas que llevan prefijos ai y ay la fuerza de voz siempre recae en ellos; estos morfemas dominan a la sílaba directa y a la mixta, incluso al fonema hache. A continuación los ejemplos de estos prefijos en las siguientes parasintéticas:

- El ai: aiwawanra. aiklaklabra, aisasara, aimamakra;

- El ay: ayulbra, ayimplikra, ayunsabra, ayinra, ayutbra;

\section{Las compuestas}

Las palabras compuestas se caracterizan por llevar dos lexemas, las cuales resultan de la unión de dos vocablos de estructura simple. En éstas, el acento prosódico del segundo vocablo se desaparece quedándose solamente con el del primero.

Los ejemplos que se presentan son palabras cuya fuerza de voz recae en la primera sílaba tónica:

-kuiwitingni ( kuiwi + tingni);

- lamadusa (lama + dusa);

- ipritingni ( ipri + tingni);

- maniwatla (mani + watla)

- utlamahta ( utla + mahta);

A continuación los ejemplos de palabras compuestas que acentúan en la segunda sílaba tónica:

- kisálaya (kisá + laya);

- awásbila ( awás +bila);

- murúbila (murú + bila);

- unúhmihta (unúh + mihta);

- kaliltara ( kalíla + tara ); 


\section{CONCLUSIONES}

En base a los resultados del estudio llegamos a las siguientes conclusiones:

- La ortografía acentual del idioma miskitu se caracteriza por disponer de un sistema de acentuación el cual se crea dos categorías de acentuación en que involucran a la primera y a la segunda sílaba tónica.

- Las vocales largas (â. İ, û,) producen sonidos prolongados al estructurarse en la sílaba mixta y algunas inversa para llevar el acento prosódico y se posesionan solamente en la primera sílaba tónica. Es incorrecto aplicar el acento ortográfico o sea el circunflejo en la sílaba directa.

- El triptongo se posesiona en la primera sílaba tónica para determinar el acento fonético; en el léxico del miskitu no se registran la presencia de este elemento lingüístico en la segunda sílaba tonal.

- El prefijo ai determina el acento prosódico en las formas verbales conjugadas que resultan de los verbos reflexivos y en las palabras parasintéticas que construyen de éstos.

- El prefijo ay (que se pronuncia ae), determina el acento prosódico de las parasintéticas que construyen de los verbos que inician con vocales y sílaba inversa tales como apaia, ikaia, ulaia, atkaia, implikaia y ulbaia.

- L a sílaba inversa determina el acento prosódico sin oposición alguna; la cual solamente se posesiona en la primera sílaba tonal.

- La sílaba mixta se ubica en la primera y en la segunda categoría acentual para determinar el acento fonético; se presentan algunas excepciones cuando en la sílaba inicial de la palabra se ubica los morfemas ai, ay, la sílaba inversa y la letra hache.

- La presencia del grafema h en ambas sílabas tónicas determina el acento prosódico sin oposición alguna.

- En las parejas de palabras homógrafas el acento prosódico se distribuye entre la primera y la segunda sílaba tónica.

- El diptongo se presenta en la primera y en la segunda sílaba tónica para determinar el acento prosódico; ante los prefijos ai y ay, y el fonema $\mathbf{h}$, se abdica.

- El acento prosódico de las palabras derivadas se hereda de la progenitora, bien puede que ésta lleve el prosódico en la primera sílaba tónica o en la segunda, pero se mantendrá la acentuación de la primitiva.

- Las parasintéticas son sustantivos derivativos, cuyo progenitor es un verbo; la acentuación de éstas lleva en la segunda sílaba tónica cuando no proceden de verbos reflexivos. 
- El acento prosódico de las palabras compuestas se desaparece el del segundo vocablo; el del primero puede ser que lo lleve en la primera o en segunda tónica, pero durante el proceso de composición se mantiene.

\section{BIIBLIOGRAFÍA}

Castell de Dueñas Beatriz (1995). Curso Básico de GRAMATICA ESPAÑOLA. Primera edición; Impreso en México.

Carreter Fernando Lázaro (1982).Teoría y Practica de la Lengua 8. Ediciones ANAYA, S.A.

Freeland Jane (2003). Lengua-Primera. Managua: Terra Nuova.

Martínez Webster Ethel (1995). Introducción al Estudio de la Lengua Miskito. Editorial Ciencias Sociales, Managua.

Matus Lazo Róger (1998). Español I. Impresión: Impresiones y Troqueles, S.A. Managua,

Nicaragua.

Moreno Cabrera Juan Carlos (2000). La dignidad e Igualdad de las Lenguas. Alianza Editorial, S.A., Madrid.

Norwood Susan. Gramática Miskita (1985). Primera Edición, CIDCA.

Peña Hernández Enrique (1991). Castellano Básico, III Curso. Sexta Edición. Managua, Nicaragua, C.A.

Salamanca Danilo (2000). Gramática Escolar del Miskito/Manual de Gramática del Miskito-Tegucigalpa.

Smutko Fray Gregorio (1996). La Presencia Capuchina entre los Miskitos- 1915-1995. Primera Edición, Cartago: Imprenta A.G. Covao.

Urbina Mancada Miguel (2004). Gramática Pedagógica Miskitu. Impresión: Multigrafic, Managua.

Vaughan Warman Adolfo (1960). Diccionario Trilingüe (miskito, español e inglés). Iglesia Católica, Waspam.

Wilson John F (1990). Obra Morava de Nicaragua /Trasfondo y Breve Historia. Impreso: Talleres Tipográficos de Editorial Unión Cardoza y CIA. LTDA. Managua, Nicaragua. 